\title{
A 10Gb/s APD-based linear burst-mode receiver with 31dB dynamic range for reach-extended PON systems
}

\author{
Xin Yin, ${ }^{1, *}$ Bart Moeneclaey, ${ }^{1}$ Xing-Zhi Qiu, ${ }^{1}$ Jochen Verbrugghe, ${ }^{1}$ Koen Verheyen, ${ }^{1}$ \\ Johan Bauwelinck, ${ }^{1}$ Jan Vandewege, ${ }^{1}$ Mohand Achouche, ${ }^{2}$ \\ and Y. Chang \\ ${ }^{1}$ Ghent University, INTEC/IMEC, Sint-Pietersnieuwstraat 41, 9000 Gent, Belgium \\ ${ }^{2}$ III-V Lab, a joint lab of Alcatel-Lucent Bell Labs, Thales R\&T and CEA Leti, Route de Nozay, 91460 Marcoussis, \\ France. \\ ${ }^{3}$ Vitesse Semiconductor Corporation, Transport Systems Engineering, 741 Calle Plano, Camarillo, CA, USA. \\ xin.yin@intec.ugent.be
}

\begin{abstract}
We present for the first time a high performance APD-based linear burst-mode receiver (BM-RX) with a record wide dynamic range of 31dB. The APD multiplication factor is controlled from burst to burst within 60ns by an on-chip self-generated M-control signal. In the experiment, a total optical budget of $54 \mathrm{~dB}$ is achieved with a single SOA-based reach extender for $10 \mathrm{~Gb} / \mathrm{s}$ upstream. With electronic dispersion compensation, the APD linear BM-RX with a wide dynamic range relaxes the requirement on optical components and lowers the system cost, especially at the optical network unit (ONU) side.
\end{abstract}

(C)2012 Optical Society of America

OCIS codes: (060.2360) Fiber optics links and subsystems; (060.0060) Fiber optics and optical communication.

\section{References and links}

1. N. Yoshimoto, "Recent R\&D trends in broadband optical access system technologies towards the secondgeneration FTTH era in Japan,” in Proceedings of Progress in Electromagnetics Research Symposium, (Marrakesh, Morocco, 2011), pp. 520-523.

2. B. Zhu, D. Au, F. Khan, and Y. Li, “1:n 10G-PON overlay of GPON link using bidirectional Raman amplifier,” in Proceedings of European Conference Optical Communication, (2012), Paper Tu.1.B.1.

3. S. Ihara, S. Yoshima, D. Mita, M. Noda, M. Nogami, and J. Nakagawa, "Over 60km transmission extended reach 10G-EPON system with a wide 29dB dynamic range burst-mode receiver,” in Proceedings of European Conference Optical Communication, (2012), Paper Tu.1.B.4.

4. H. Bulow, "Electronic equalization of transmission impairments," in Proceedings of Optical Fiber Communication Conference and Exhibit (Optical Society of America, 2002), pp. 24-25, Anaheim, Calif, USA.

5. P. Ossieur, N. A. Quadir, S. Porto, M. Rensing, C. Antony, W. Han, P. O. Brien, Y. Chang, and P. D. Townsend, "A 10G linear BMRX supporting electronic dispersion compensation for extended-reach optical links,” in Proceedings of European Conference Optical Communication, (2011), Paper Th.13.B.4.

6. P. Ossieur, N. A. Quadir, S. Porto, M. Rensing, C. Antony, P. O’Brien, Y (Frank) Chang, and P. D. Townsend, "A 10G linear burst-mode receiver supporting electronic dispersion compensation for extended-reach optical links, ” Opt. Express, 19, 604 (2011).

7. T. Nakanishi, Y. Fukuda, K. Suzuki, N. Yoshimoto, M. Nakamura, K. Kato, K. Nishimura, Y. Ohtomo, and M. Tsubokawa, "Wide dynamic range and high sensitivity APD burst receiver configuration based on M-switching technique for 10GEPON system," in Proceedings of Lasers and Electro-Optics Society (Institute of Electrical and Electronics Engineers, 2007), Paper ThW5.

8. X. Yin, X.Z. Qiu, J. Gillis, J. Put, J. Verbrugghe, J. Bauwelinck, J. Vandewege, H. Krimmel, D. van Veen, P. Vetter, and F. Chang, "Experiments on $10 \mathrm{~Gb} / \mathrm{s}$ fast settling high sensitivity burst-mode receiver with on-chip auto-reset for 10G-GPONs [Invited],” J. of Opt. Communications and Networking, 4, B68-B76 (2012).

9. X. Yin, B. Moeneclaey, X.Z. Qiu, J. Verbrugghe, K. Verheyen, J. Bauwelinck, J. Vandewege, M. Achouche, and F.Y. Chang, "A 10Gb/s APD-based linear burst-mode receiver with 31dB dynamic range for reachextended PON systems," in Proceedings of European Conference Optical Communication, (2012), Paper Tu.1.B.5. 
10. “ITU-T Recommendation G.987.2: 10-Gigabit-capable passive optical networks (XG-PON): Physical media dependent (PMD) layer specification,” (ITU-T, 2010).

11. Y.F. Chang, "Recent progress of EDC commercialization in addressing datacom and telecom challenges to enable high-speed optical enterprise, metro and long-haul networks," in Proceedings of Optical Fiber Communication Conference/National Fiber Optic Engineers Conference, OSA Technical Digest (Optical Society of America, 2007), paper NWA2.

12. X. Yin, J. Put, J. Verbrugghe, J. Gillis, X.-Z. Qiu, J. Bauwelinck, J. Vandewege, H.-G. Krimmel and M. Achouche, "A 10Gb/s burst-mode TIA with on-chip reset/lock CM signaling detection and limiting amplifier with a 75ns settling time,” in Proceedings of Solid-State Circuits Conference Digest of Technical Papers (ISSCC), (2012), paper 24.4.

\section{Introduction}

Passive optical network (PON) systems are being widely deployed as a most cost-effective solution for optical access networks. Some recent research focused on upgrading existing PON systems with extended reach and increased splitting ratio [1-3]. Extending the link reach enables rural subscriber services while maintaining the PON's cost benefits, and increasing the splitting ratio allows offering services to urban subscribers with reduced number of optical line terminals (OLTs).

Although the majority of existing OLTs employ a burst-mode receiver (BM-RX) with a limiting amplifier, the link reach can be further extended using an APD-based linear BM-RX, which allows the use of electronic equalization techniques [4]. As the link reach is extended, the possible difference between upstream burst signal intensities from different optical network units (ONUs) is also increased. Therefore, not only high receiver sensitivity but also a wide dynamic range is of an important consideration for the requirement of the APD linear $\mathrm{BM}-\mathrm{RX}$. The APD-based solution allows the linear BM-RX to operate without an optical preamplifier and the bias-dependent multiplication factor (M) adds another degree of freedom to increase the linear operation range. The 10Gb/s PIN-based linear BM-RX in $[5,6]$ had a dynamic range of $22.7 \mathrm{~dB}$. In the final experimental setup, an erbium doped fiber amplifier (EDFA) before the PIN-based linear BM-RX was used as a pre-amplifier. Reference [7] described a 10Gb/s limiting-type BM-RX using an external signal to control the APD's multiplication factor (M-control) and achieved a dynamic range of 24.4dB. Recently, we have demonstrated a high performance limiting-type BM-RX supporting multi-rate (2.5G/5G/10Gb/s) upstream with more than 26.3dB dynamic range without using APD Mcontrol [8].

In this paper, we report, for the first time, an APD-based linear burst-mode receiver with a dynamic range of $31 \mathrm{~dB}$, by using an on-chip self-generated M-control signal [9]. In addition, the proposed APD-based linear BM-RX technique is also intended to relax the requirement on optical components and lower the system cost, especially at ONU side. Our experiment shows that a lower bandwidth/performance optical transmitter can be used at the ONU for $10 \mathrm{~Gb} / \mathrm{s}$ upstream transmission with the proposed APD linear BM-RX. The remainder of the paper is organized as follows. The realization of the APD linear BM-RX and APD bias circuit are discussed in details in Section 2. Section 3 and Section 4 present the experimental setup and results. We finally conclude in Section 5.

\section{Realization of the APD linear BM-RX with self-generated M-control signal}

Figure 1 shows the proposed 10Gb/s APD linear BM-RX architecture. The linear BM-RX consists of a transimpedance amplifier (TIA) stage, a variable gain amplifier (VGA), and a linear output buffer. At the start of each burst, the fast automatic gain control (AGC) senses the output amplitude of the TIAc core and set its transimpedance gain within 10ns. After the transimpedance gain is stabilized, the output amplitude of the TIAc core is measured again by the AGC block. Using this second amplitude measurement result, the M-control block generates a digital M-control signal to the APD bias circuit. In this way, the APD bias voltage (and consequently its multiplication factor) can be adjusted accordingly to a smaller value 
when the BM-RX receives a very strong incoming burst signal. A DC-offset compensation block sets the balanced signal to keep the amplifier circuit working near the middle of the linear range.

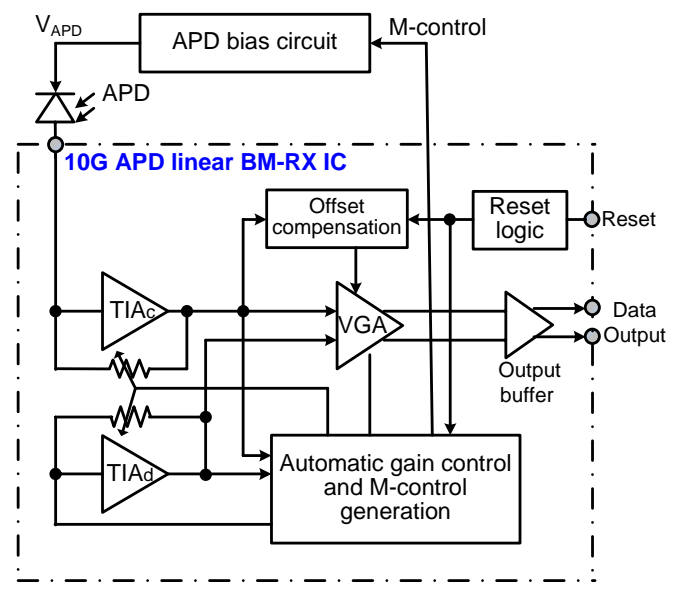

Fig. 1. APD linear BM-RX architecture

The simplified schematic of the APD bias circuit is shown in Fig. 2(a). $R_{1}, R_{2}$ and nMOSFET $M_{1}$ form an inverter with the output voltage switching from $V_{C C}$ to $V C C \cdot\left(R_{2}+R_{O N}\right) /\left(R_{1}+R_{2}+R_{O N}\right)$, where $R_{O N}$ is the on-resistance of the analog switch $M_{1}$. The output drive stage is an opamp $A_{2}$ configured as a voltage amplifier with feedback resistors $R_{3}$ and $R_{4}$, providing a voltage gain of $1+R_{3} / R_{4}$. Between the inverter and the output drive stage, an amplifier $A_{1}$ serves as a voltage buffer due to a relatively high output impedance of the inverter. The decoupling design of the APD bias voltage $V_{A P D}$ is now becoming more important because we are varying the APD's M-factor to increase the linear range of the BM-RX. As shown in Fig. 2(a), the parasitic inductance $L_{B}$ is mainly due to the bonding wire and module lead and the value is usually small. A small capacitance $C_{1}$ has been integrated inside the APD BM-RX during the module assembly, which can decouple the $V_{A P D}$ properly if only $L_{B}$ is there. Another important but often overlooked, parasitic inductance should be taken into account is the $L_{A}$, composed of the lead inductance of the output drive stage and the PCB strip inductance. Because APD is a temperature sensitive device, in the design we keep the hot spot, e.g. the output drive stage $A_{2}$, far away from the APD. The SPICE simulation (Fig. 2(b)) shows that the M-control signal will cause significant ringing on the $V_{A P D}$ when a long PCB trace is present. Therefore, an additional decoupling capacitor $C_{P C B}$ is used to tune out the effect of the $L_{A}$, and this reduces the settling time of APD's M-factor considerably.

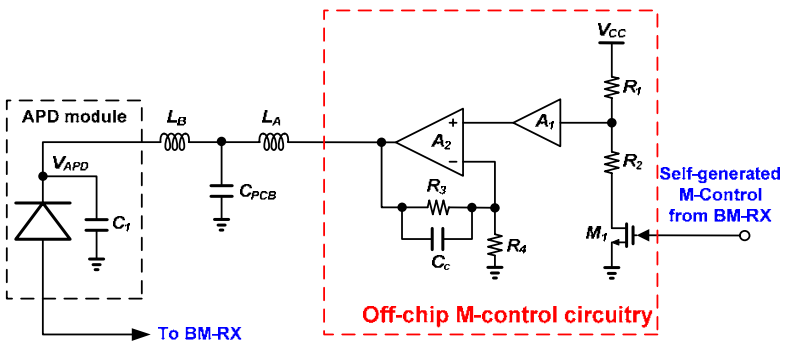

(a)

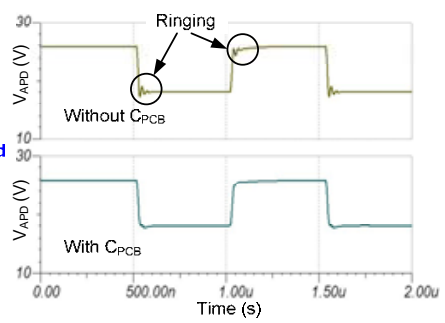

(b)

Fig. 2. (a) Off-chip M-control circuitry for APD biasing, (b) simulated APD bias voltage without and with $C_{P C B}$ 
Inside the BM-RX, the entire amplifier chain was designed for a high linearity (no internal hard decisions) for the whole operating range. After each burst, the linear BM-RX is reset to its highest gain and the APD multiplication factor is restored to the default high value. The simulated total harmonic distortion (THD) as a function of input optical power is shown in Fig. 3. The THD has been simulated with a pure sine wave with optical power swept from $-32 \mathrm{dBm}$ to $+0.5 \mathrm{dBm}$ (input frequency was $250 \mathrm{MHz}$ and extinction ratio was $8 \mathrm{~dB}$ ). The improvement achieved by the M-control scheme (APD M was switched from 6.1 to 2.7, and APD responsivity at $\mathrm{M}=1$ was $0.8 \mathrm{~A} / \mathrm{W}$ ) has been also evaluated with the simulation. In case that the maximum distortion threshold of $10 \%$ was assumed, more than $3 \mathrm{~dB}$ enhancement for the achievable linear range was found as illustrated in Fig. 3. There are two reasons to schedule the APD M-control after the TIAc core gain adjustment. Firstly, compared to the APD M-switching settling time ( 60ns), adjusting TIAc core gain is faster and more predictable in terms of settling time, which simplifies the timing requirement between the fast AGC and the M-control procedure. Secondly, the APD actually behaves as a current gain stage ahead of the TIAc core. Therefore, for sufficiently high burst signal levels, the gain in the later stage (TIAc core in this case) should be reduced before reducing the gain in the earlier stage (APD M-factor) in order to assure a better signal to noise ratio.

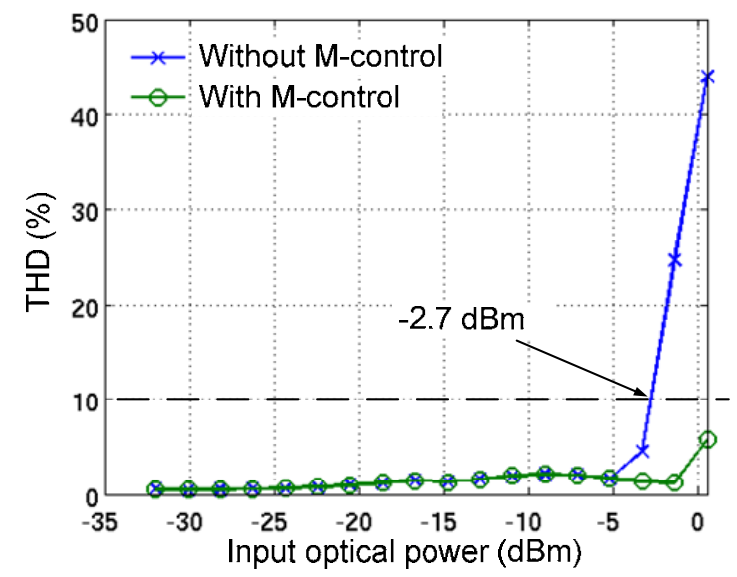

Fig. 3. Simulated THD versus input optical power with and without M-control.

\section{Experimental setup}

The 10Gb/s APD-based linear BM-RX IC was fabricated in a $0.13 \mu \mathrm{m}$ SiGe BiCMOS process. The linear BM-RX IC occupies $1.3 \times 1 \mathrm{~mm}^{2}$ and consumes $250 \mathrm{~mW}$ from a single $2.7 \mathrm{~V}$ power supply. Figure 4 shows the experimental setup. The reach extender used a single semiconductor optical amplifier (SOA) with a small signal gain of $20 \mathrm{~dB}$ and a noise figure of $6.2 \mathrm{~dB}$. The SOA's peak gain wavelength was $1290 \mathrm{~nm}$ and saturation output power was around $10.6 \mathrm{dBm}$. A $10 \mathrm{~nm}$ coarse wavelength division multiplexing (CWDM) filter was used to remove out-of-band amplified spontaneous emission (ASE) noise from the SOA. The setup also includes $10 \mathrm{~km}+10 \mathrm{~km}$ optical fibers in the access and feeder sections respectively. Variable optical attenuators (VOAs) were used to vary the access and feeder budget. 


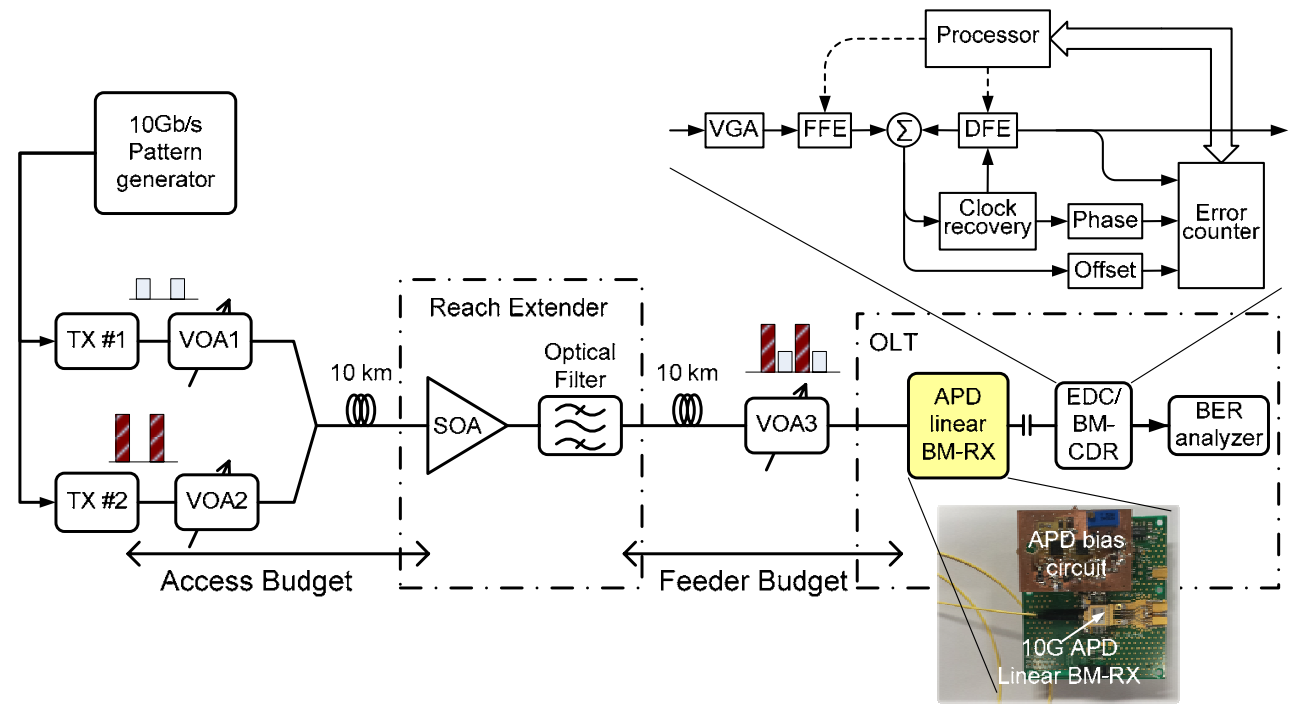

Fig. 4. Experimental setup for performance evaluation of the APD linear BM-RX for reachextended PON systems

\section{Experimental results}

The APD-based linear BM-RX was initially characterized in a back-to-back (B2B) configuration without fibers and the reach extender. The $10 \mathrm{~Gb} / \mathrm{s}$ burst consisted of a 150ns preamble of 1010 pattern (including the settling time for APD M-control, linear BM-RX and BM-CDR) and a 1280ns payload. The guard time between consecutive bursts was 51.2ns. Two $1.3 \mu \mathrm{m}$ burst-mode transmitters (BM-TXs) were used to send 10Gb/s upstream bursts: TX\#1 consists of a laser externally modulated using an electro-absorption modulator and TX\#2 is a DFB laser based TOSA. The power level provided by TX\#2 was set at $-7 \mathrm{dBm}$ at the input of the APD-based linear BM-RX, while only the bit error ratio (BER) of the packet from TX\#1 was recorded. Figure 5(a) plots the measured received burst signals, and selfgenerated M-control digital signal. As shown in Fig. 5(b), the APD bias voltage $V_{A P D}$ was adjusted between 25.2V ( $\mathrm{M}=6.1)$ for weak bursts and 18V $(\mathrm{M}=2.7)$ for strong bursts. The rise and fall time of the APD bias voltage switching was 40ns and 35ns respectively. In this experiment, the bias voltage switching range was chosen conservatively to avoid any possible APD breakdown. In a production-level implementation, an additional control circuit for the APD bias voltage can be used to compensate the temperature shift and therefore a more aggressive switching range can be obtained to further extend the linear range of the BM-RX.

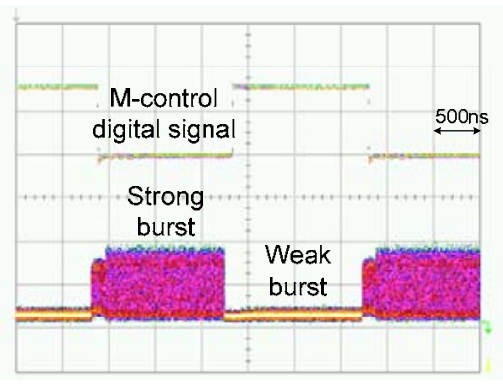

(a)

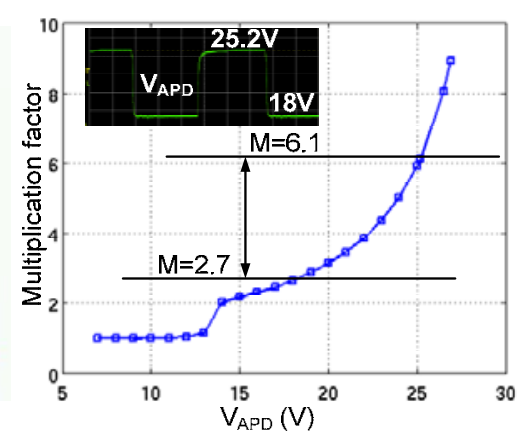

(b)

Fig. 5. (a) Incoming burst and M-control waveform (b) APD bias voltage waveform and the corresponding $\mathrm{M}$ value 
Figure 6 shows the measured BERs with and without M-control. When M-control is disabled, the measured RX sensitivity was $-30.5 \mathrm{dBm}$ and the overload was $-2.2 \mathrm{dBm}$ at a pre forward error correction (FEC) BER of $10^{-3}$. After enabling the M-control, the input sensitivity at pre-FEC BER of $10^{-3}$ remains unchanged while the measured overload improves to $+0.5 \mathrm{dBm}$. This yields an optical dynamic range of $31 \mathrm{~dB}$.

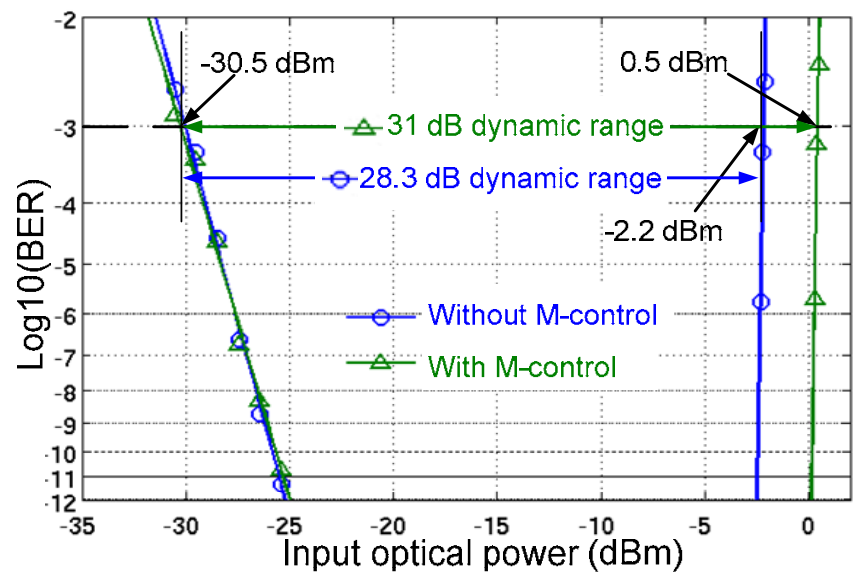

Fig. 6. Measured B2B BER curves in BM operation with and without M-control

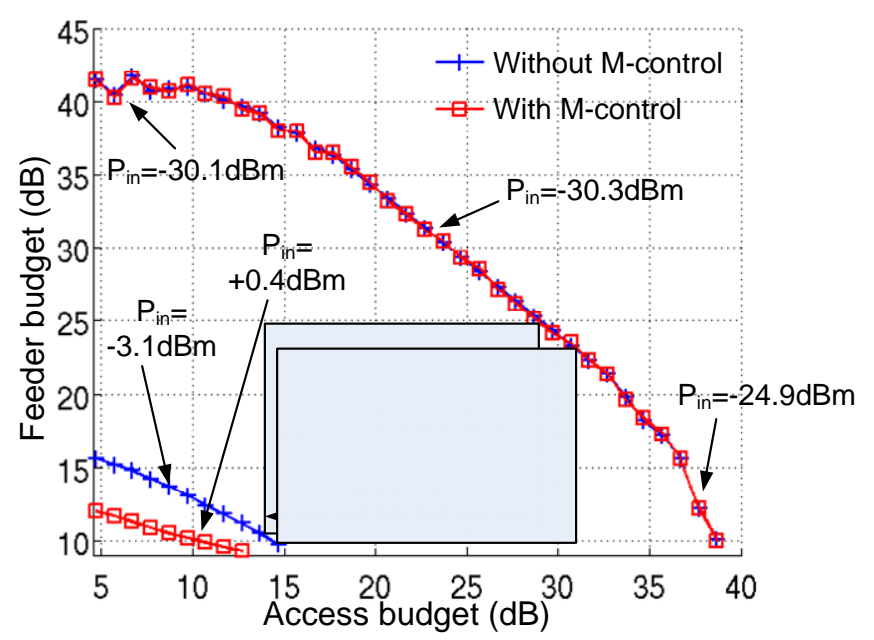

Fig. 7. Measured BER $\left(B E R=10^{-3}\right)$ contour with and without $M$-control for reach extended $10 \mathrm{~Gb} / \mathrm{s}$ upstream.

To evaluate the APD linear BM-RX performance in the single SOA-based reach extender configuration, we measured the constant-BER contour over the access and feeder budget plane. Figure 7 shows the measured BER contour at $B E R=10^{-3}$ with and without $\mathrm{M}$-control. In both cases, a feeder budget of $23 \mathrm{~dB}$ (or $24.9 \mathrm{~dB}$ ) is allowed while the access budget is within the optical distribution network (ODN) class N2 (or N1) [10]. The total obtainable optical budget in either case is about $54 \mathrm{~dB}$ (with TX\#1 power $+4.4 \mathrm{dBm}$ ). In addition, the M-control scheme enlarges the admissible area in the access and feeder plane, enabling the possibility for new ODN specifications. 


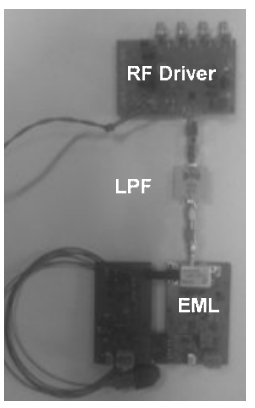

(a)

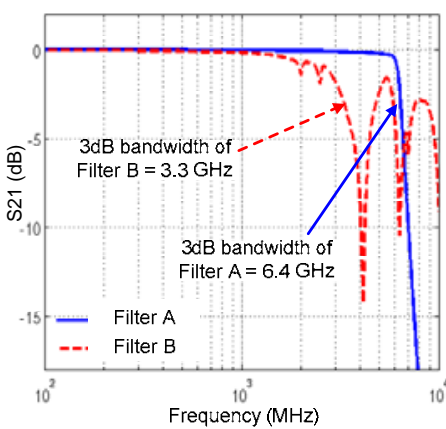

(b)
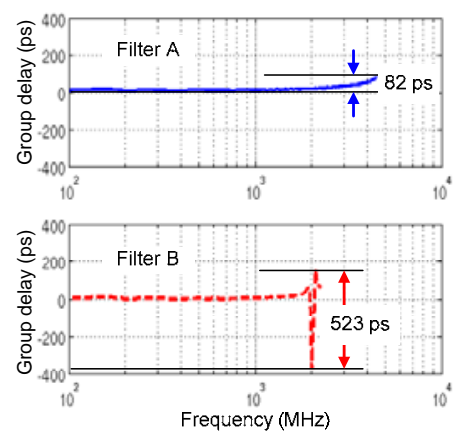

(c)

Fig. 8. (a) Optical TX configuration (b) measured S21 parameter of the LPF (c) measured group delay of the LPF

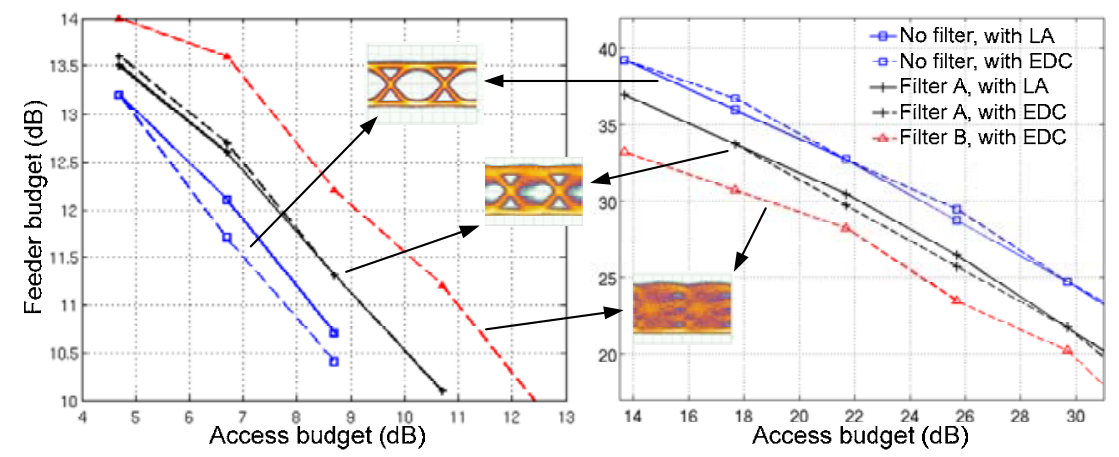

Fig. 9. Comparison of linear and limiting-type BM-RX for different bandwidth optical TXs.

We finally validated the linearity of the APD linear BM-RX by enabling the electronic dispersion compensation (EDC) in the BM-CDR chip. The EDC consists of 7-stage feedforward equalization (FFE) and 4-stage decision feedback equalization (DFE) [11]. The EDC tap coefficients were optimized for one burst and then fixed in the burst-mode experiment, as no EDC chip on burst basis was available. M-control has also been enabled in the experiments with EDC. In PON systems, the fiber dispersion for upstream transmission is less of a problem as the standard single-mode fiber has nearly zero dispersion around the $1.3 \mu \mathrm{m}$ wavelength. Instead of compensating the transmission impairments due to the fibers, we used the electronic equalization to compensate the impairments caused by the low bandwidth optical TX at the ONU. This allows for cost-effective components to be used in the ONU for $10 \mathrm{~Gb} / \mathrm{s}$ upstream transmission. As shown in Fig. 8(a), an electronic low-pass filter (LPF) was inserted between the RF driver and the EML in TX\#1 to emulate a low bandwidth ONU TX. The TX bandwidth has been estimated from its step response. The estimated bandwidths for no filter, with filter $\mathrm{A}$ and with filter $\mathrm{B}$ cases, are $10,6.3$, and $3 \mathrm{GHz}$ respectively. The measured S21 parameter and group delay of the LPFs are shown in Fig. 8(b) and Fig. 8(c) respectively. To emulate a low performance ONU TX, the filter B has been designed with not only a small bandwidth $(\sim 3.3 \mathrm{GHz})$, but also a large group delay variation of 523ps within the pass-band (75\% of the $3 \mathrm{~dB}$ bandwidth). Figure 9 shows the measured TX eye diagram and $\mathrm{BER}=10^{-3}$ contour with linear or limiting-type BM-RX. A BM limiting amplifier (LA) [12] was used with the linear BM-RX to build a limiting-type BM-RX. When the ONU TX had reasonably good output eye-diagram (i.e., $6.3 \mathrm{GHz}$ and $10 \mathrm{GHz} \mathrm{TX}$ cases), the limiting-type and linear BM-RXs achieved similar performance. However, the linear BM-RX and EDC/CDR chip are able to recover the upstream signal with significant inter-symbol 
interference (ISI), while the limiting-type BM-RX failed to meet the pre-FEC BER threshold for the $3 \mathrm{GHz}$ TX case.

\section{Conclusions}

We have demonstrated for the first time a 10Gb/s APD-based linear BM-RX for reachextended PON systems. Using an on-chip self-generated M-control signal, it achieved 31dB dynamic range. In conjunction with an EDC/CDR chip, the APD linear BM-RX with a wide dynamic range of $31 \mathrm{~dB}$ enables flexible network configurations and relaxes the performance requirements on low-cost ONU optical components.

\section{Acknowledgements}

The authors thank the EU-funded FP7 ICT projects EURO-FOS, Agency for Innovation by

Science and Technology in Flanders (IWT), and Sumitomo Electric Devices Innovations, Inc for providing EML and APD-TIA ROSA assembly. 